\title{
BMJ Open Evaluating the efficacy of Internet-Based Exercise programme Aimed at Treating knee Osteoarthritis (iBEAT-OA) in the community: a study protocol for a randomised controlled trial
}

\author{
Sameer Akram Gohir, ${ }^{1}$ Paul Greenhaff, ${ }^{1,2,3,4,5}$ Abhishek Abhishek, ${ }^{1,6}$ Ana M. Valdes ${ }^{1}$
}

To cite: Gohir SA, Greenhaff P, Abhishek A, et al. Evaluating the efficacy of Internet-Based Exercise programme Aimed at Treating knee 0steoarthritis (BEAT-OA) in the community: a study protocol for a randomised controlled trial. BMJ Open 2019;9:e030564. doi:10.1136/ bmjopen-2019-030564

- Prepublication history and additional material for this paper are available online. To view these files, please visit the journal online (http://dx.doi org/10.1136/bmjopen-2019030564).

Received 20 March 2019 Revised 22 July 2019 Accepted 17 September 2019

Check for updates

(c) Author(s) (or their employer(s)) 2019. Re-use permitted under CC BY. Published by BMJ.

For numbered affiliations see end of article.

Correspondence to

Sameer Akram Gohir;

sameer.gohir@nottingham.ac.uk

\section{ABSTRACT}

Introduction Knee osteoarthritis $(0 A)$ is the most common joint disease worldwide. As of today, there are no diseasemodifying drugs, but there is evidence that muscle strengthening exercises can substantially reduce pain and improve function in this disorder, and one very well tested physiotherapy protocol is the 'Better Management of Patients with Osteoarthritis' developed in Sweden. Given the high prevalence of knee $0 \mathrm{~A}$, a potentially costeffective, digitally delivered approach to treat knee $0 \mathrm{~A}$ should be trialled. This study aims to explore the benefits of iBEAT-OA (Internet-Based Exercise programme Aimed at Treating knee 0steoarthritis) in modulating pain, function and other health-related outcomes in individuals with knee $O A$.

Methods and analysis A randomised controlled trial was designed to evaluate the efficacy of a web-based exercise programme in a population with knee 0A compared with standard community care provided by general practitioners (GPs) in the UK. We anticipate recruiting participants into equal groups. The intervention group $(n=67)$ will exercise for 20-30 min daily for six consecutive weeks, whereas the control group ( $n=67$ ) will follow GP-recommended routine care. The participants will be assessed using a Numerical Rating Scale, the Western Ontario and McMaster Universities 0steoarthritis Index, the Arthritis Research UK Musculoskeletal Health Questionnaire, the Pittsburgh Sleep Quality Index, 30 s sit to stand test, timed up and go test, quantitative sensory testing, musculoskeletal ultrasound scan, muscle thickness assessment of the vastus lateralis, and quadriceps muscles force generation during an isokinetic maximum voluntary contraction (MVC). Samples of urine, blood, faeces and synovial fluid will be collected to establish biomarkers associated with changes in pain and sleep patterns in individuals affected with knee $0 A$. Standard parametric regression methods will be used for statistical analysis.

Ethics and dissemination Ethical approval was obtained from the Research Ethics Committee (ref: 18/EM/0154) and the Health Research Authority (protocol no: 18021). The study was registered in June 2018. The results of the trial will be submitted for publication in a peer-reviewed journal.

Trial registration number NCT03545048
Strengths and limitations of this study

- This study is the first to evaluate a web-based exercise intervention to improve pain in sufferers of knee osteoarthritis $(\mathrm{OA})$ in the UK.

- We plan to recruit only those individuals presenting with radiographic evidence of knee $0 \mathrm{~A}$ that will rule out non-OA causes of knee pain.

- This study is the first to evaluate changes in sleep disturbance due to knee $\mathrm{OA}$ along with an exercise intervention.

- The lack of double-blind study design is typically a limitation of exercise and lifestyle intervention studies.

- Six weeks of iBEAT-0A (Internet-Based Exercise programme Aimed at Treating knee Osteoarthritis) intervention may not show a statistically detectable reduction in knee pain and may be a potential limitation of this study.

\section{INTRODUCTION}

Knee osteoarthritis (OA) is a common cause of disability globally and is mostly managed in primary care. ${ }^{1}$ In the UK, $10 \%$ of people between the ages of 65 and 74 consult their general practitioners (GPs) for OA every year. ${ }^{2}$ In the UK, 1 in 25 people consult their GP for knee OA annually. ${ }^{1}$

The first line of treatment for OA involves exercise, education and weight loss if applicable. $^{3-7}$ Exercise improves joint and patient-centred outcomes in knee $\mathrm{OA}$, and encourages them to manage activities of daily living. ${ }^{8-21}$ In fact, strengthening knee exercises have been shown to reduce progression of radiographic changes of knee $\mathrm{OA}^{22} \mathrm{~A}$ recent Cochrane review on exercise interventions for knee OA has reported that exercise significantly reduced pain (12 points/100; 95\% CI 10 to 15$)$ and improved physical function (10 points/100; 95\% CI 8 to 13) 
to a moderate degree. ${ }^{23}$ Another systematic review and meta-analysis conducted on the effect of resistance exercises in patients with knee OA reported that resistance training relieved pain (standard mean difference: -0.43 ; $95 \% \mathrm{CI}-0.57$ to -0.29$).{ }^{24}$ There is a disparity of opinion regarding the effectiveness of different types of exercise for knee OA to reduce pain, and a combination of open and closed isotonic exercises is recommended for knee OA. ${ }^{25}$ Symptoms of knee arthritis can be exacerbated by following ineffective or unsafe exercises regimens, ${ }^{26-29}$ leading to poor prognosis and poor adherence to exercise intervention, ${ }^{30}$ hence the need to choose the type and frequency of exercise intervention carefully.

Versus Arthritis recommends knee exercise for 'knee pain'; however, these exercises are not specific to knee $\mathrm{OA}$ and include soft tissue injuries of knee as a cause of knee pain. ${ }^{31}$ An exercise regimen is normally recommended by GPs as part of the first line of treatment when they consult someone with arthritis-related knee pain. If an exercise intervention fails to impact positively on pain perception, patients are referred to physiotherapy. After a comprehensive assessment by physiotherapists, a variety of exercise interventions that fluctuate in exercise modality, intensity and duration can be prescribed, but with variable success in terms of outcome. Therefore, there is a need for standardised exercise interventions to address knee OA pain, which would ideally be accessible online to maximise efficacy and cost-effectiveness. iBEAT-OA (Internet-Based Exercise programme Aimed at Treating knee Osteoarthritis) is the first randomised controlled trial (RCT) conducted on the app-based knee exercises developed by Joint Academy (JA). The exercise intervention (JA App), which we will use in this project, is a web-based programme derived from the Supported Osteoarthritis Self-Management Programme (SOASP) also known as the 'Better Management of Patients with Osteoarthritis' (BOA) developed in Sweden. ${ }^{32}$ Essentially, the JA App is a digital version of face-to-face BOA. From January 2008 until January 2017, around 75000 patients participated in the SOASP, 2339 physiotherapists and occupational therapists were educated to deliver the SOASP, and today SOASP is offered in 700 clinics all over Sweden. ${ }^{32}$ This intervention has been rated good to very good by $94 \%$ of patients at reducing pain related to knee $\mathrm{OA}$ and has an excellent safety profile and acceptability. ${ }^{33}$ A quasi-experimental study done on the internet-based JA programme has shown a change in mean Numerical Rating Scale (NRS) score which was larger than the minimal clinical difference (5.4 vs $4.1 ; \mathrm{p}<0.001){ }^{34}$

There exist a number of other exercise programmes within care programmes for people with knee OA pain. Among these is the Good Life with osteoArthritis in Denmark (GLA:D) programme, which is a registry-based study that implemented clinical guidelines (patient education and exercise but not weight loss) to patients with knee OA. ${ }^{35}$ The programme consists of a 2-day training course for physiotherapists, including training to diagnose and deliver OA care, and an 8-week supervised exercise intervention for patients with OA, with a minimum intervention of three sessions in total. However, GLA:D does not deliver intervention via a web or smartphone app.

The ESCAPE (Enabling Self-management and Coping with Arthritic Pain using Exercise) app is a support tool for people who have already attended a person-toperson ESCAPE programme ${ }^{18}$ and enables participants to continue to exercise safely in the home environment following the person-to-person programme. A limitation of this approach is that the app is a support tool for the main programme and provides reminders for exercise. It cannot be used as the sole basis for treatment and care of knee OA. The recommendation is to use it in conjunction with the advice and professional judgement of the GP or other healthcare practitioners, which is the limitation of this app.

Because of the high prevalence of knee OA, strategies to deliver exercise interventions that are both efficacious and inexpensive should be prioritised. Some of the issues relating to the delivery of exercise interventions for knee OA include compliance, accessibility to clinics for people with mobility problems and the cost of delivering such services. There are previous studies which have assessed the efficacy of home-based exercises ${ }^{10} 1236$; however, very few studies looked at web-based delivery of exercise intervention. ${ }^{34}$ 37-42 Most of these studies recruited patients with knee pain, and only two studies assessed for radiographic evidence of knee OA. ${ }^{3941}$ This makes the results of these studies less specific to base a recommended treatment for knee OA. In addition, none of these studies has investigated the role of exercise interventions for pain relief, assessing levels of pain sensitivity and central sensitisation, which are likely different for knee OA and other causes (eg, soft tissue injuries) of knee pain.

The most recent RCT which studied the benefit of internet-based exercises versus routine physiotherapy reported no difference on the Western Ontario and McMaster Universities Osteoarthritis Index (WOMAC) at 12 months between internet-based versus face-to-face physiotherapy, and suggested further studies with strategies to maximise the benefit of exercises-based interventions for patients with knee OA. ${ }^{41}$ Therefore, there is a need for specific, efficacious and cost-effective exercise programme that individuals can perform in the home setting.

Knee OA pain is accompanied by a number of additional disturbances that influence the individual's health, which we also propose to study. There is a well-known link between sleep disturbance and chronic pain, and epidemiological studies ${ }^{43-45}$ and experimental studies ${ }^{46-50}$ have established a link between disturbed sleep and knee OA. These studies suggest that individuals with OA have greater sleep disturbances. Furthermore, sleep disturbance is recognised as an important factor in determining pain perception. ${ }^{50} \mathrm{~A}$ relationship between sleep disturbance and pain severity in patients with knee OA is usually explored, and sleep disturbances such as shortened sleep duration and fragmented sleep ${ }^{43}$ have been 


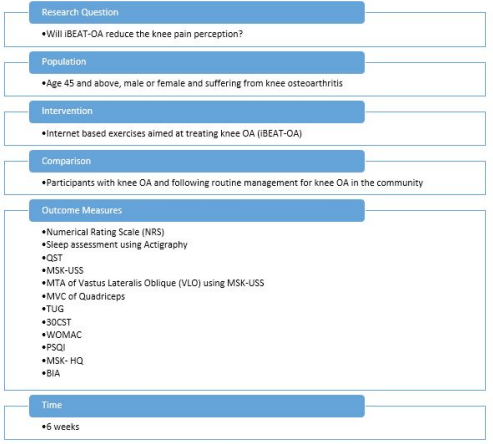

Figure 1 A randomised controlled trial evaluating the efficacy of Internet-Based Exercise programme Aimed at Treating knee Osteoarthritis (iBEAT-OA)-PICOT format. 30CST, 30 s sit to stand test; BIA, bioimpedance analysis; MSK-HQ, Arthritis Research UK Musculoskeletal Health Questionnaire; MSK-USS, musculoskeletal ultrasound; MTA, muscle thickness assessment; MVC, maximum voluntary contraction - isokinetic contraction of the quadriceps muscle; OA, osteoarthritis; PSQI, Pittsburgh Sleep Quality Index; QST, quantitative sensory testing, TUG, timed up and go test; WOMAC, Western Ontario and McMaster Universities Osteoarthritis Index.

associated with increased sensitivity to pain in patients with $\mathrm{OA}$, and consequently with decreased quality of life. ${ }^{45}$ Therefore, studying the sleeping pattern is highly relevant if we are discussing a successful online exercise programme for knee OA.

Recent epidemiological and clinical studies have also underlined that metabolic syndrome has the most significant impact on the initiation and severity of OA. ${ }^{51-55}$ Metabolic-triggered inflammation, also known as meta-inflammation, ${ }^{56}$ can be a result of abnormalities in body composition, adipokines, cytokines, lipids and vitamin D and has been associated with the pathogenesis of OA. ${ }^{54}$ Given that body composition is influenced by a number of the variables associated with OA, for example, systemic inflammation, sleep patterns, altered physical activity levels and altered energy levels, it is important to study this as well. The intricate links between exercises, sleep, pain, metabolic syndrome, body composition and OA are not fully understood. To date, the relationship between improvements in pain due to exercise for pain relief and other changes related to health parameters that have been linked to OA or chronic pain has not been explored. Specifically, the effects of exercise for knee pain relief on sleep, biomarkers of inflammation and insulin resistance are unknown. We aim to study these parameters and establish the link between exercises for knee OA and these parameters. Figure 1 shows this in PICOT format.

The efficacy of web-based delivery of exercises has not been evaluated in a UK setting. This is the first study to conduct web-based exercises intervention in the UK. This study is also novel because it will be the first in the UK to bring together a wide range of factors influencing knee $\mathrm{OA}$. This will include quantitative sensory testing (QST), which is deemed to be an objective measure, along with measurement of sleep patterns undertaken using an activity monitor (actigraphy).

\section{Rationale}

This study aims to explore the benefits of an internet-based exercise programme in patients with knee OA to establish if 6-week intervention reduces pain perception and sensitivity. Being a web-based exercise intervention also makes it accessible and cost-effective to volunteers and physiotherapists, and will hopefully help establish a standardised intervention for knee $\mathrm{OA}$ that will maintain individual motivation, compliance and managing pain. The study will also endeavour to explore the complicated relationship between chronic pain, sleep, biomarkers of inflammation, body composition and knee OA. Therefore, this study could potentially help generate recommendations for the treatment of knee OA.

\section{Primary objective}

The primary objective is to test whether an internet-based exercise intervention can reduce pain perception (NRS) in knee OA.

\section{Secondary objective}

The secondary objective is to test whether there is a benefit of iBEAT-OA in improving sleep disturbances and in reducing pain sensitisation and metabolic syndrome.

\section{METHODS AND ANALYSIS \\ iBEAT-OA trial design}

The iBEAT-OA study is an RCT in the primary care setting in Nottingham with participants identified as having knee OA, randomised 1:1 to web-based exercises or usual care, as shown in figure 2 .

\section{Setting}

The study will be in a home-based, primary care setting.

\section{Patient and public involvement}

At the patient and public involvement (PPI) representative meeting on 14 December 2015, seven volunteers suffering from chronic OA pain were asked about the relevance of studying sleep in the context of OA pain. They all were very supportive of studying and understanding sleep patterns. They viewed actigraphy as a good non-invasive alternative to polysomnography and saw no problem with using the device for 6 weeks.

On 17 May 2017, six representatives from the PPI musculoskeletal group were asked about exercise interventions and were all supportive of this. They were also asked about the extraction of synovial fluid from their joints. Five out of six said they would not have a problem with this if it was performed by a specialist using ultrasound to guide the needle. This approach was therefore adopted as an optional test within the study.

The latest version of the patient facing documentation (participant information sheet, consent form, invitation 


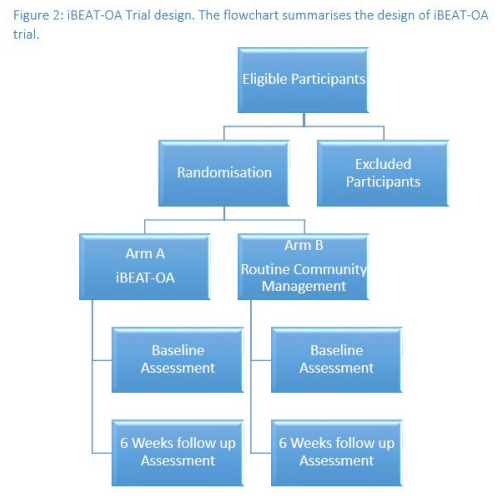

Figure 2 iBEAT-OA trial design. The flow chart summarises the design of iBEAT-OA trial. iBEAT-OA, Internet-Based Exercise programme Aimed at Treating knee Osteoarthritis.

letter and recruitment flyer) have been shared with the PPI group and received favourable comments.

\section{Recruitment}

A selection of eligible people for the study will be invited from existing databases ${ }^{57}$ held at Academic Rheumatology, City Hospital Nottingham of participants with knee pain who have agreed to be contacted for future studies. The inclusion and exclusion criteria are shown in figure 3. Any shortfall in the recruitment will be compensated by sending study leaflets to GP surgeries. The GPs will follow the inclusion and exclusion criteria. All those individuals who are suitable for the study will be sent the study information sheet. Those who return the completed screening consent form will be contacted and screened for inclusion in the study.

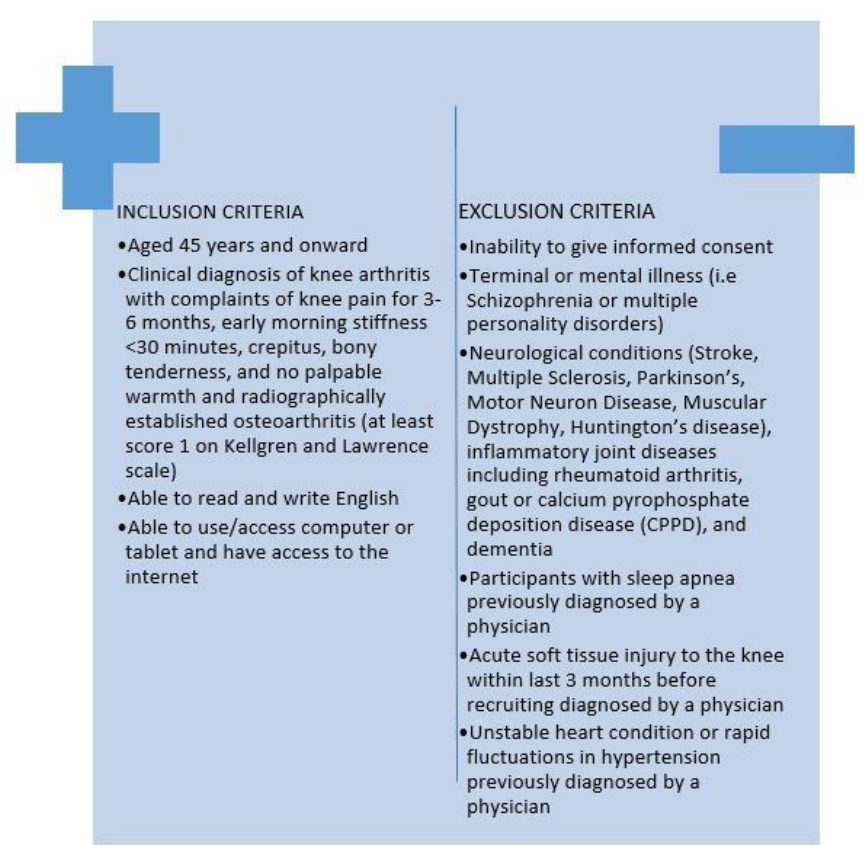

Figure 3 Inclusion and exclusion criteria for iBEAT-OA trial. iBEAT-OA, Internet-Based Exercise programme Aimed at Treating knee Osteoarthritis.

\section{Randomisation and blinding}

Eligible participants will undergo computer-generated randomisation and allocation concealment. Randomisation will be done using 'sealed envelope' (https://www. sealedenvelope.com/), an online randomisation service. Participants will be equally allocated between treatment arms with at least $n=67$ per arm and this will be done by the research team. At this point, individuals will be alerted to which study group they have been assigned, and hence blinding is not possible. Because this might affect the motivation of participants randomised into the control arm, these individuals will be offered access to the web-based exercise programme after completing the study. Blinding of exercise intervention studies is difficult; however, the clinical research team will be blinded to the intervention.

\section{Sample size and justification}

A Swedish study using specifically JA as the intervention and NRS as the outcome reported an effect size of 1.3 on the NRS, ${ }^{34}$ which corresponds to 0.56 SD (average SD 2.3). A study with an expected 60 participants per arm at the end of the 6-week intervention has $86 \%$ power to achieve such an effect size with an alpha of 0.05 . However, a recent systematic review of 44 high-quality exercise trials for knee OA pain (3537 participants ${ }^{23}$ found an average effect of 12/100 Visual Analogue Scale (VAS) points corresponding to $0.49 \mathrm{SD}$. A sample size of $\mathrm{n}=60$ per group is necessary to achieve $75 \%$ statistical power. The estimated dropout rate for exercise interventions is $10 \%$; therefore, a sample of 67 per arm will be recruited to achieve between $75 \%$ and $86 \%$ power, that is, $80 \%$ power with an alpha level of 0.05 .

\section{Intervention}

iBEAT-OA will use a web-based exercise platform known as JA, as recent pilot studies ${ }^{34} 40$ demonstrated promising results. This programme is based on a Swedish face-to-face self-management programme known as 'Artrosskolan' (The Osteoarthritis School), which provided structured information and exercises for knee OA to a relevant population suffering from knee OA. This was initially introduced as 'Better Management of Patients with Osteoarthritis ${ }^{58}$ Compliance to this self-management programme has been reported as good. Of 20200 patients, $62 \%$ reported daily use of this programme at 3-month follow-up; however, this percentage drops to $37 \%$ at 12-month review. ${ }^{33}$ The company that produced the JA platform has given permission to use their web-based app to conduct this study.

The exercise intervention comprises a mixture of open and closed chain exercise manoeuvres, and a combination of concentric and eccentric exercise modalities, and is focused on the overall strength of legs including the muscles around the hips and knee joints. An open kinetic chain is defined as 'a combination of successively arranged joints in which the terminal segments can move freely' ${ }^{59}$ Closed chain exercises are exercises or movements where the distal aspect of the extremity is fixed to an object that is stationary and proximal joints move.$^{59}$ There are 
balance enhancement exercises as well. There are educational sessions integrated into the programme covering the basics of OA, its treatment, self-managing symptoms of $\mathrm{OA}$ and the benefits of maintaining a healthy lifestyle.

\section{Control group}

The control group will continue with routine self-management or GP management of knee OA which is offered in the community setting in the UK.

\section{Follow-up duration}

Duration of follow-up will be 6 weeks postintervention.

The 6-week duration has been selected for this study based on a previous observational quasi-experimental study which reported 6 weeks of web-based treatment were effective to reduce knee pain. ${ }^{34}$

\section{Research assessment}

Outcome Measures in Rheumatology-Osteoarthritis Research Society International has recommended six domains as mandatory to be measured and reported in all hip and knee OA clinical trials. ${ }^{60}$ These are pain, physical function, quality of life, patient's global assessment of the target joint, adverse events including mortality, and joint structure (in specific circumstances and depending on the intervention). The first four domains are measured by NRS, QST, WOMAC and Arthritis Research UK Musculoskeletal Health Questionnaire (MSK-HQ). The adverse events will be reported at the end of the trial, and joint structure will be reported based on X-rays and the findings of ultrasound scan of the most painful knee. Time up and go test and $30 \mathrm{~s}$ sit to stand test (30CST) are objective assessments of physical functions based on the recommendations of Osteoarthritis Research Society International $^{61}$; thus, the following outcome measures will be assessed:

- Sleep.

- NRS (average pain on the day of assessment on a scale of $0-10)$.

- QST (pressure pain threshold, temporal summation and conditional pain modulation).

- Inflammatory markers on ultrasound (synovial fluid, synovial hypertrophy and hypervascularity), using musculoskeletal ultrasound scan (MSK-USS).

- Muscle thickness assessment (MTA) of vastus lateralis oblique using MSK-USS.

- Maximum voluntary contraction (MVC) - isokinetic contraction of the quadriceps muscle.

- Biomarkers of insulin resistance.

- Physical functioning (time up and go test, 30CST).

- WOMAC.

- Pittsburgh Sleep Quality Index (PSQI).

- General health questionnaire (MSK-HQ).

- Body composition assessment by bioimpedance analysis.

\section{Start and end dates}

The trial has started in February 2019 and enrolment will end in December 2020.

\section{Description of intervention}

Participants seeking care for OA will be informed of the study both orally and in writing. Those who qualify for the study will give signed informed consent, and the participants will be randomised to an intervention or control group. The consent will be taken by an experienced member of the research team. The exception applies to those participants who have not had knee X-rays in the previous 12 months. These individuals will be called to the hospital, and after gaining valid consent knee radiographs will be obtained and their X-rays will be assessed by experienced staff. Once a definite eligibility criterion (Kellgren and Lawrence (K/L) score at least 1 or above) is established in such cases, the qualifying participants will be randomised to the intervention or control group.

The intervention group will have an assessment session with experienced staff, and NRS, ${ }^{62-64}$ WOMAC, ${ }^{65} 66$ MSK-HQ ${ }^{6768} \mathrm{PSQI},{ }^{69-72}$ 30CST, ${ }^{73-75}$ timed up and go test (TUG), ${ }^{77}$ QST, ${ }^{78-84}$ MSK-USS, ${ }^{85-89}$ MTA of vastus lateralis, ${ }^{90} 91$ quadriceps muscle force generation during an isokinetic MVC, ${ }^{92}$ and urine and blood samples ${ }^{93} 94$ will be taken at baseline (refer to online supplementary file for further information). These samples will be assessed for circulating levels of fasting insulin, glucose, $\mathrm{C}$ reactive protein, triglycerides, low-density lipoprotein, high-density lipoprotein, tumour necrosis factor-alpha, interleukin-6 and interleukin-1. Those who consent for aspiration of synovial fluid will go through an ultrasound-guided aspiration procedure. ${ }^{85-88}$ The intervention group will be given an actigraphy device (ActTrust). Their sleeping pattern will be recorded quantitatively.

The intervention group will then receive a link via email, which will be used to $\log$ in to the JA online portal (see https://www.jointacademy.com). After log-in has been achieved, the exercise intervention will start. This will consist of a 6-week internet-based physical therapy programme that will provide information, exercise, contact details of a personal physiotherapist, education about lifestyle and behavioural changes. This intervention can be accessed using a smartphone or a tablet. The programme also encourages physical activity adherence by sending email prompts on a regular basis. Initially, participants will answer an online questionnaire covering areas such as joint pain intensity, health-related quality of life, physical function, as well as performing a physical test assessing lower limb strength. This questionnaire will form part of the online baseline assessment. The exercise intervention will consist of knee and hip exercises along with some functional activities such as sit to stand and stairs climbing.

After the 6-week exercise intervention, participants will fill in the same questionnaire and perform the same physical tests to enable evaluation. There are two face-toface meetings between participants and physiotherapist/ nurse, at enrolment and after 6 weeks. The physiotherapist will be available via asynchronous online chat or over the phone during the 6-week study period. 
The control group will continue with their routine self-management which is offered in the community set-up. They will be assessed on NRS, QST, WOMAC, MSK-HQ, PSQI, 30CST, TUG, MSK-USS, MTA of vastus lateralis, MVC of the quadriceps muscle, body composition, and urine and blood samples at baseline. The control group will also use actigraphy to monitor sleep patterns. They will follow the routine management of knee OA recommended by the National Institute for Health and Care Excellence (NICE), which includes non-pharmacological and pharmacological management. ${ }^{95}$ They will be reassessed after 6 weeks on the same outcome measures to determine if there has been an impact of self-management strategies.

\section{Data management}

In the iBEAT-OA trial, data will be collected during the first and last session. Additional, weekly pain scores will be collected via an online portal, and actigraphy will be used to collect sleep patterns. Online portal and actigraphy data will be collated by trained local research staff, and data entry in a relational Microsoft Access database will be completed in a standardised fashion. The clinical research forms from the first and last study visit will be sent to the data entry site. A central data manager performs and monitors data entry. This will include questionnaires (MSK-HQ, PSQI, WOMAC) and data from QST, 30CST, TUG, MSK-USS, MTA of vastus lateralis, body composition and MVC of the quadriceps muscle.

\section{Statistical analysis}

Clinical trial data will be analysed using an intention-totreat approach. We will compare outcome measures (eg, pain sensitivity, pain scores, sleep patterns and inflammatory measures) between the exercise and non-exercise groups controlling for baseline scores using appropriate parametric and non-parametric statistical tests. Additional observational secondary analyses (ie, correlations between change in sleep patterns and change in pain measures) will be carried out using parametric statistics and adjusting for the relevant covariates. These missing data will be calculated based on multiple imputation. The SPSS package will be used for statistical analyses.

\section{Adverse events}

There are no serious adverse events reported with these exercises. These exercises have been trialled on 75000 patients from 2008 to 2017 with no serious adverse events. ${ }^{33} 58$ There is a small chance of an increase in the knee, hip or back pain. ${ }^{23}$ We will monitor the pain levels of the patient on a weekly basis using an internet-based interface to monitor any increase in the knee, hip or low back pain. If the pain exacerbates to the level that the participant starts struggling with the activities of daily living, then they will be advised to stop participating in the study and to contact their GP.

\section{Criteria for terminating the study}

As the study involves only two assessments and does not involve investigational medicinal products or medical devices, and the same intervention has not given rise to any serious adverse events in over 70000 participants in Sweden, it is not envisaged that circumstances will arise that require termination of this study.

\section{Ethics and dissemination}

The study results will be submitted to Versus Arthritis, regulatory authorities and a peer-reviewed journal for publication. Also, the results will be presented at national and international conferences. Study participants will also be informed of the results if requested.

\section{Insurance and indemnity}

Insurance and indemnity for clinical study participants and study staff are covered within the National Health Service (NHS) Indemnity Arrangements for clinical negligence claims in the NHS, issued under the cover of Health Service Guidelines HSG (96)48. There are no special compensation arrangements, but study participants may have recourse through the NHS complaints procedures.

The University of Nottingham as research sponsor indemnifies its staff, research participants and research protocols with both public liability insurance and clinical trials insurance. These policies include provision for indemnity in the event of a successful litigious claim for proven non-negligent harm.

\section{Study conduct}

Study conduct will be subject to systems audit for inclusion of essential documents; permissions to conduct the study; Curriculum Vitaes (CVs) of study staff and training received; local document control procedures; consent procedures and recruitment logs; adherence to procedures defined in the protocol (eg, inclusion/exclusion criteria, timeliness of visits); and accountability of study materials and equipment calibration logs.

\section{Study data and audits}

Monitoring of study data shall include confirmation of informed consent; source data verification; data storage and data transfer procedures; local quality control checks and procedures; back-up and disaster recovery of any local databases; and validation of data manipulation. This will be managed by the direct study team.

Entries on study forms will be verified by inspection against the source data. A sample of the forms (10\%) will be checked on a regular basis for verification of all entries made. In addition, the subsequent capture of the data on the study database will be checked. Where corrections are required, these will carry a full audit trail and justification.

Study data and evidence of monitoring and systems audits will be made available for inspection by the Research Ethics Committee (REC) as required. 


\section{Record retention and archiving}

In compliance with theInternational Council for Harmonisation of Technical Requirements for Pharmaceuticals for Human Use (ICH) and Good Clinical Practice (GCP) guidelines, regulations and in accordance with the University of Nottingham Code of Research Conduct and Research Ethics, the chief or local principal investigator will maintain all records and documents regarding the conduct of the study. These will be retained for at least 7 years or for longer if required. If the responsible investigator is no longer able to maintain the study records, a second person will be nominated to take over this responsibility.

The study master file held by the chief investigator on behalf of the sponsor shall be finally archived at secure archive facilities at the University of Nottingham. This archive shall include all anonymised study databases and associated meta-data encryption codes.

\section{Statement of confidentiality}

Individual participants' medical or personal information obtained as a result of this study is considered confidential, and disclosure to third parties is prohibited. Participant confidentiality will be further ensured by using identification code numbers to correspond to treatment data in the computer files. Such medical information may be given to the participant's medical team and all appropriate medical personnel responsible for the participant's welfare.

Data generated as a result of this study will be available for inspection on request by the participating physicians, University of Nottingham representatives, REC, local research and development (R\&D) departments, and regulatory authorities.

\section{Data sharing statement}

Data originating from the iBEAT-OA trial will be available on request once the results from the trial have been published in a peer-reviewed publication. Researchers interested in accessing the data will need to complete a 'Data Access Proposal Form', and the investigators associated with iBEAT-OA will grant access to the data provided these are used for research purposes only. No personal information of research participants will be shared as part of any data sharing.

\section{DISCUSSION}

Knee OA remains one of the most common forms of $\mathrm{OA}$ and affects the majority of the population in the UK. Treatments include non-pharmacological and pharmacological management recommended by NICE. The non-pharmacological management recommends local muscles strengthening, general aerobic fitness, weight loss and using transcutaneous electrical nerve stimulation as an adjunct to other forms of management. ${ }^{95}$ The majority of the population requires some guidance as to what exercises they should do and get referred to the
Versus Arthritis website for basic exercises. If those exercises fail to make much difference or if patients struggle to understand these exercises, they are referred to a local physiotherapy department in the community. This means that some of these patients will have to travel to local community health centres or hospitals to see a physiotherapist and learn the relevant exercises.

The JA or other similar platform could work in-between the Versus Arthritis leaflet and referral to physiotherapy, thus cutting the cost of travelling, and saving the time of the patient wasted in travelling and the time of physiotherapists so that they can treat other patients with more complex pathologies and needs.

Although iBEAT-OA will not specifically study the economic benefits of delivering the JA intervention instead of face-to-face physiotherapy, it will generate data on its efficacy which will serve as a starting point for any future health economic and cost-effectiveness analysis of such a method for relieving knee OA pain.

The majority of physiotherapists guide their patients on the type of exercises they should follow and review them a few times before the patients are recommended to self-manage $\mathrm{OA}$, and at this stage these patients get discharged. The compliance of patients afterwards can decline as they may stop exercising. There are various reasons for this, including adjusting lifestyle to include these exercises, pain during the exercises, lack of motivation and lack of professional monitoring. ${ }^{96-98}$ The JA platform is designed to keep these patients motivated by sending regular emails to remind them and by tracking their progress.

Social media is a powerful platform which offers a connection between users and is a source of social interaction for a range of individuals. This can be used to promote health and to treat patients with OA. ${ }^{99-101} \mathrm{JA}$ is aimed at using social media such as internet and digital application on a mobile phone to encourage patients with knee OA to self-manage their condition. This programme will educate and train them to stay 'in-control' of their knee OA, which will improve their overall quality of life. This will lead to the overall psychological well-being of our population. This study will also encourage other researchers to study digital health platform, which will be the preferred way of communication and solution to health-related issues for next generations.

This study intends to establish the link between digital exercises, muscles strength, knee inflammation, sleep disturbance, pain and severity of knee OA. The intention is to check if exercising regularly can reduce pain, knee inflammation and sleep disturbance and slow down the progression of knee OA. If this complicated link can be interpreted effectively, we may find a way to reduce the progression of knee OA.

\section{Author affiliations}

${ }^{1}$ NIHR Nottingham Biomedical Research Centre, School of Medicine, University of Nottingham, Nottingham, UK 
${ }^{2}$ Division of Physiology, Pharmacology and Neuroscience, University of Nottingham School of Medical and Surgical Sciences, Nottingham, UK

${ }^{3}$ MRC Arthritis Research UK Centre, MSK Ageing Research, Nottingham, United Kingdom

${ }^{4}$ School of Life Sciences, University of Nottingham, Nottingham, United Kingdom ${ }^{5}$ NIHR Nottingham Biomedical Research Centre, School of Medicine, University of Nottingham, Nottingham, United Kingdom

${ }^{6}$ Academic Rheumatology, University of Nottingham, Nottingham, United Kingdom

\section{Twitter Sameer Akram Gohir @sameer_vicky}

Acknowledgements We would like to thank Dr Michelle Hall (Assistant Professor, Division of Physiotherapy and Rehabilitation Sciences, University of Nottingham) and Tony Kelly (Research Metrologist, Academic Rheumatology, University of Nottingham) for their valuable contribution to designing, monitoring and implementing this study.

Contributors SG is the primary author and all other authors are secondary. AMV is the main supervisor and is leading this project. $P G$ and $A A$ are secondary supervisors. All authors have equally contributed to this article.

Funding This study is part-funded by the Pain Centre Versus UK (University of Nottingham) [Grant numbers 21960, 18769] and by Nottingham Biomedical Research Centre (BRC).

\section{Competing interests None declared.}

Patient consent for publication Not required.

Ethics approval The study has received approval from the Research Ethics Committee (REC) (ref: 18/EM/0154), Health Research Authority (HRA) (protocol no: 18021) and the Nottingham University Hospitals NHS Trust Research \& Innovation (R\&l) department (ref: 18RH004). Any modification to the approved protocol will require resubmission of modifications and further approval from the REC and the sponsor.

Provenance and peer review Not commissioned; externally peer reviewed.

Open access This is an open access article distributed in accordance with the Creative Commons Attribution 4.0 Unported (CC BY 4.0) license, which permits others to copy, redistribute, remix, transform and build upon this work for any purpose, provided the original work is properly cited, a link to the licence is given, and indication of whether changes were made. See: https://creativecommons.org/ licenses/by/4.0/.

\section{REFERENCES}

1 Peat G, McCarney R, Croft P. Knee pain and osteoarthritis in older adults: a review of community burden and current use of primary health care. Ann Rheum Dis 2001;60:91-7.

2 Royal College of General P, McCormick A, Fleming D, Charlton $\mathrm{J}$, Great B, Office of Population C, et al. Morbidity statistics from general practice: fourth national study 1991-1992. London: H.M.S.O 1995.

3 Conaghan PG, Dickson J, Grant RL. Care and management of osteoarthritis in adults: summary of NICE guidance. BMJ 2008;336:502-3.

4 Jevsevar DS. Treatment of osteoarthritis of the knee: evidencebased guideline, 2nd edition. J Am Acad Orthop Surg 2013;21:571-6.

5 Pendleton Aet al. EULAR recommendations for the management of knee osteoarthritis: report of a task force of the standing Committee for international clinical studies including therapeutic trials (ESCISIT). Ann Rheum Dis 2000;59:936-44.

6 Bennell KL, Hunter DJ, Hinman RS. Management of osteoarthritis of the knee. BMJ 2012;345:e4934.

7 Hochberg MC, Altman RD, April KT, et al. American College of rheumatology 2012 recommendations for the use of nonpharmacologic and pharmacologic therapies in osteoarthritis of the hand, hip, and knee. Arthritis Care Res 2012;64:465-74

8 Bennell KL, Hinman RS. A review of the clinical evidence for exercise in osteoarthritis of the hip and knee. J Sci Med Sport 2011;14:4-9.

9 Messier SP, Loeser RF, Miller GD, et al. Exercise and dietary weight loss in overweight and obese older adults with knee osteoarthritis: the arthritis, diet, and activity promotion trial. Arthritis Rheum 2004;50:1501-10.
10 Thomas KS, Muir KR, Doherty M, et al. Home based exercise programme for knee pain and knee osteoarthritis: randomised controlled trial. BMJ 2002;325:752.

11 van Baar ME, Assendelft WJ, Dekker J, et al. Effectiveness of exercise therapy in patients with osteoarthritis of the hip or knee: a systematic review of randomized clinical trials. Arthritis Rheum 1999;42:1361-9.

12 Chamberlain MA, Care G, Harfield B. Physiotherapy in osteoarthrosis of the knees: a controlled trial of hospital versus home exercises. Int Rehabil Med 1982;4:101-6.

13 Ettinger WH, Burns R, Messier SP, et al. A randomized trial comparing aerobic exercise and resistance exercise with a health education program in older adults with knee osteoarthritis. The fitness arthritis and seniors trial (fast). JAMA 1997;277:25-31.

14 Kovar PA, Allegrante JP, MacKenzie CR, et al. Supervised fitness walking in patients with osteoarthritis of the knee. A randomized, controlled trial. Ann Intern Med 1992;116:529-34.

15 Minor MA, Webel RR, Kay DR, et al. Efficacy of physical conditioning exercise in patients with rheumatoid arthritis and osteoarthritis. Arthritis Care Res. 1989;32:1396-405.

16 Fisher NM, Pendergast DR, Gresham GE, et al. Muscle rehabilitation: its effect on muscular and functional performance of patients with knee osteoarthritis. Arch Phys Med Rehabil 1991;72:367-74.

17 Pisters MF, Veenhof C, van Meeteren NLU, et al. Long-Term effectiveness of exercise therapy in patients with osteoarthritis of the hip or knee: a systematic review. Arthritis Rheum 2007:57:1245-53

18 Hurley MV, Walsh NE, Mitchell HL, et al. Clinical effectiveness of a rehabilitation program integrating exercise, self-management, and active coping strategies for chronic knee pain: a cluster randomized trial. Arthritis Rheum 2007;57:1211-9.

19 Dunlop DD, Song J, Semanik PA, et al. Physical activity levels and functional performance in the osteoarthritis initiative: a graded relationship. Arthritis Rheum 2011;63:127-36.

20 Esser S, Bailey A. Effects of exercise and physical activity on knee osteoarthritis. Curr Pain Headache Rep 2011;15:423-30.

21 Lange AK, Vanwanseele B, Fiatarone singh MA. Strength training for treatment of osteoarthritis of the knee: a systematic review. Arthritis Rheum 2008;59:1488-94.

22 Mikesky AE, Mazzuca SA, Brandt KD, et al. Effects of strength training on the incidence and progression of knee osteoarthritis. Arthritis Rheum 2006;55:690-9.

23 Fransen M, McConnell S, Harmer AR, et al. Exercise for osteoarthritis of the knee: a Cochrane systematic review. $\mathrm{Br} \mathrm{J}$ Sports Med 2015;49:1554-7.

$24 \mathrm{Li} \mathrm{Y}, \mathrm{Su} \mathrm{Y}, \mathrm{Chen} \mathrm{S}$, et al. The effects of resistance exercise in patients with knee osteoarthritis: a systematic review and metaanalysis. Clin Rehabil 2016;30:947-59.

25 Baker K, McAlindon T. Exercise for knee osteoarthritis. Curr Opin Rheumatol 2000;12:456-63.

26 Raske A, Norlin R. Injury incidence and prevalence among elite weight and power lifters. Am J Sports Med 2002;30:248-56.

27 Reeves RK, Laskowski ER, Smith J. Weight training injuries. Phys Sportsmed 1998;26:67-96.10.3810/psm.1998.02.939

28 Reeves RK, Laskowski ER, Smith J. Weight training injuries. Phys Sportsmed 1998;26:54-73.

29 Escamilla RF, Fleisig GS, Zheng N, et al. Effects of technique variations on knee biomechanics during the squat and leg press. Medicine \& Science in Sports \& Exercise 2001:33:1552-66.

30 Peeler J, Ripat J. The effect of low-load exercise on joint pain, function, and activities of daily living in patients with knee osteoarthritis. Knee 2018;25:135-45.

31 UK AR. Exercises to manage knee pain. Available: https://www. arthritisresearchuk.org/arthritis-information/exercises-to-managepain/knee-pain-exercises.aspx

32 BOA. Better management of patients with osteoarthritis (BOA). Available: https://boa.registercentrum.se/boa-in-english/bettermanagement-of-patients-with-osteoarthritis-boa/p/By_o8GxVg

33 Thorstensson CA, Garellick G, Rystedt H, et al. Better management of patients with osteoarthritis: development and nationwide implementation of an evidence-based supported osteoarthritis self-management programme. Musculoskeletal Care 2015;13:67-75.

34 Nero H, Dahlberg J, Dahlberg LE. A 6-week web-based osteoarthritis treatment program: observational quasi-experimental study. J Med Internet Res 2017;19:e422.

35 Skou ST, Roos EM. Good Life with osteoArthritis in Denmark (GLA:DTM): evidence-based education and supervised neuromuscular exercise delivered by certified physiotherapists nationwide. BMC Musculoskelet Disord 2017:18:72. 
36 Talbot LA, Gaines JM, Huynh TN, et al. A home-based PedometerDriven walking program to increase physical activity in older adults with osteoarthritis of the knee: a preliminary study. J Am Geriatr Soc 2003:51:387-92.

37 Lorig KR, Ritter PL, Laurent DD, et al. The internet-based arthritis self-management program: a one-year randomized trial for patients with arthritis or fibromyalgia. Arthritis Rheum 2008;59:1009-17.

38 Bossen D, Veenhof C, Van Beek KE, et al. Effectiveness of a webbased physical activity intervention in patients with knee and/or hip osteoarthritis: randomized controlled trial. J Med Internet Res 2013;15:e257.

39 Brooks MA, Beaulieu JE, Severson HH, et al. Web-Based therapeutic exercise resource center as a treatment for knee osteoarthritis: a prospective cohort pilot study. BMC Musculoskelet Disord 2014;15:158.

40 Dahlberg LE, Grahn D, Dahlberg JE, et al. A web-based platform for patients with osteoarthritis of the hip and knee: a pilot study. JMIR Res Protoc 2016;5:e115

41 Allen KD, Arbeeva L, Callahan LF, et al. Physical therapy vs Internetbased exercise training for patients with knee osteoarthritis: results of a randomized controlled trial. Osteoarthritis and Cartilage 2018;26:383-96.

42 Bennell KL, Nelligan R, Dobson F, et al. Effectiveness of an internetdelivered exercise and Pain-Coping skills training intervention for persons with chronic knee pain: a randomized TriallnternetDelivered exercise and Pain-Coping skills training for knee pain. Annals of internal medicine 2017;166:453-62.

43 Wilcox S, Brenes GA, Levine D, et al. Factors related to sleep disturbance in older adults experiencing knee pain or knee pain with radiographic evidence of knee osteoarthritis. J Am Geriatr Soc 2000;48:1241-51.

44 Power JD, Perruccio AV, Badley EM. Pain as a mediator of sleep problems in arthritis and other chronic conditions. Arthritis Rheum 2005;53:911-9.

45 Smith MT, Quartana PJ, Okonkwo RM, et al. Mechanisms by which sleep disturbance contributes to osteoarthritis pain: a conceptual model. Curr Pain Headache Rep 2009;13:447-54.

46 Leigh TJ, Hindmarch I, Bird HA, et al. Comparison of sleep in osteoarthritic patients and age and sex matched healthy controls. Ann Rheum Dis 1988:47:40-2.

47 Doherty M, Smith J. Elusive 'alpha-delta' sleep in fibromyalgia and osteoarthritis. Ann Rheum Dis 1993;52:245

48 Harrison MM, Childs A, Carson PE. Incidence of undiagnosed sleep apnea in patients scheduled for elective total joint arthroplasty. $J$ Arthroplasty 2003;18:1044-7.

49 Association ASD. International classification of sleep disorders. revised: diagnostic and coding manual, 1997. Available: http://www absm org/PDF/ICSD pdf

50 Campbell CM, Buenaver LF, Finan P, et al. Sleep, pain Catastrophizing, and central sensitization in knee osteoarthritis patients with and without insomnia. Arthritis Care Res 2015;67:1387-96.

51 Gosset M, Berenbaum F, Salvat C, et al. Crucial role of visfatin/ pre-B cell colony-enhancing factor in matrix degradation and prostaglandin E2 synthesis in chondrocytes: possible influence on osteoarthritis. Arthritis Rheum 2008;58:1399-409.

52 Sowers M, Karvonen-Gutierrez CA, Palmieri-Smith R, et al. Knee osteoarthritis in obese women with cardiometabolic clustering. Arthritis Rheum 2009;61:1328-36.

53 Puenpatom RA, Victor TW. Increased prevalence of metabolic syndrome in individuals with osteoarthritis: an analysis of NHANES III data. Postgrad Med 2009;121:9-20.

54 Wang X, Hunter D, Xu J, et al. Metabolic triggered inflammation in osteoarthritis. Osteoarthritis Cartilage 2015;23:22-30.

55 Zhuo Q, Yang W, Chen J, et al. Metabolic syndrome meets osteoarthritis. Nat Rev Rheumatol 2012;8:729-37.

56 Gregor MF, Hotamisligil GS. Inflammatory mechanisms in obesity. Annu Rev Immunol 2011;29:415-45.

57 Fernandes GS, Sarmanova A, Warner S, et al. Knee pain and related health in the community study (KPIC): a cohort study protocol. BMC Musculoskelet Disord 2017;18:404

58 Academy J. Better management of patients with osteoarthritis -BOA, 2008. Available: https://boa.registercentrum.se/boa-inenglish/better-management-of-patients-with-osteoarthritis-boa/p/ By_08GxVg

59 GJ ETD. Closed kinetic chain exercise: a comprehensive guide to multiple joint exercise. Human Kinetics Publishers, 2001: 128.

60 Smith TO, Hawker GA, Hunter DJ, et al. The OMERACT-OARSI core domain set for measurement in clinical trials of hip and/or knee osteoarthritis. J Rheumatol 2019:jrheum.181194.
61 Dobson F, Hinman RS, Roos EM, et al. OARSI recommended performance-based tests to assess physical function in people diagnosed with hip or knee osteoarthritis. Osteoarthritis Cartilage 2013;21:1042-52.

62 Ferreira-Valente MA, Pais-Ribeiro JL, Jensen MP. Validity of four pain intensity rating scales. Pain 2011;152:2399-404.

63 Hjermstad MJ, Fayers PM, Haugen DF, et al. Studies comparing numerical rating scales, verbal rating scales, and visual analogue scales for assessment of pain intensity in adults: a systematic literature review. J Pain Symptom Manage 2011;41:1073-93.

64 Karcioglu O, Topacoglu H, Dikme O, et al. A systematic review of the pain scales in adults: which to use? Am J Emerg Med 2018;36:707-14.

65 McConnell S, Kolopack P, Davis AM. The Western Ontario and McMaster universities osteoarthritis index (WOMAC): a review of its utility and measurement properties. Arthritis \& Rheumatism 2001;45:453-61.

66 Bellamy N, Buchanan WW, Goldsmith CH, et al. Validation study of WOMAC: a health status instrument for measuring clinically important patient relevant outcomes to antirheumatic drug therapy in patients with osteoarthritis of the hip or knee. J Rheumatol 1988:15:1833-40.

67 Hill JC, Kang S, Benedetto E, et al. Development and initial cohort validation of the arthritis research UK musculoskeletal health questionnaire (MSK-HQ) for use across musculoskeletal care pathways. BMJ Open 2016;6:e012331.

68 Hill J, Kang S, Benedetto E, et al. Development of the musculoskeletal health questionnaire (MSK-HQ) for use in different conditions and different healthcare pathways. Value in Health 2016;19.

69 Backhaus J, Junghanns K, Broocks A, et al. Test-Retest reliability and validity of the Pittsburgh sleep quality index in primary insomnia. J Psychosom Res 2002:53:737-40.

70 Buysse DJ, Reynolds CF, Monk TH, et al. Quantification of subjective sleep quality in healthy elderly men and women using the Pittsburgh sleep quality index (PSQI). Sleep 1991;14:331-8.

71 Carpenter JS, Andrykowski MA. Psychometric evaluation of the Pittsburgh sleep quality index. J Psychosom Res 1998;45:5-13.

72 Finan PH, Buenaver LF, Bounds SC, et al. Discordance between pain and radiographic severity in knee osteoarthritis: findings from quantitative sensory testing of central sensitization. Arthritis \& Rheumatism 2013;65:363-72.

73 Gill S, McBurney H. Reliability of performance-based measures in people awaiting joint replacement surgery of the hip or knee. Physiotherapy Research International 2008;13:141-52.

74 Gill SD, de Morton NA, Mc Burney H. An investigation of the validity of six measures of physical function in people awaiting joint replacement surgery of the hip or knee. Clin Rehabil 2012;26:945-51.

75 Jones CJ, Rikli RE, Beam WC. A 30-S chair-stand test as a measure of lower body strength in community-residing older adults. Res $Q$ Exerc Sport 1999;70:113-9.

76 Bennell K, Dobson F, Hinman R. Measures of physical performance assessments: Self-Paced Walk Test (SPWT), Stair Climb Test (SCT), Six-Minute Walk Test (6MWT), Chair Stand Test (CST), Timed Up \& Go (TUG), Sock Test, Lift and Carry Test (LCT), and Car Task. Arthritis Care Res 2011;63:S350-70.

77 Podsiadlo D, Richardson S. The Timed "Up \& Go": A Test of Basic Functional Mobility for Frail Elderly Persons. J Am Geriatr Soc 1991;39:142-8.

78 Arendt-Nielsen L, Nie H, Laursen MB, et al. Sensitization in patients with painful knee osteoarthritis. Pain 2010;149:573-81.

79 Finan PH, Buenaver LF, Bounds SC, et al. Discordance between pain and radiographic severity in knee osteoarthritis: findings from quantitative sensory testing of central sensitization. Arthritis Rheum 2013;65:363-72.

80 Imamura M, Imamura ST, Kaziyama HHS, et al. Impact of nervous system hyperalgesia on pain, disability, and quality of life in patients with knee osteoarthritis: a controlled analysis. Arthritis Rheum 2008:59:1424-31.

81 Lee YC, Lu B, Bathon JM, et al. Pain sensitivity and pain reactivity in osteoarthritis. Arthritis Care Res 2011;63:320-7.

82 Neogi T, Frey-Law L, Scholz J, et al. Sensitivity and sensitisation in relation to pain severity in knee osteoarthritis: trait or state? Ann Rheum Dis 2015;74:682-8.

83 Neogi T, Guermazi A, Roemer F, et al. Association of joint inflammation with pain sensitization in knee osteoarthritis: the multicenter osteoarthritis study. Arthritis Rheumatol 2016;68:654-61.

84 Wessel $\mathrm{J}$. The reliability and validity of pain threshold measurements in osteoarthritis of the knee. Scand J Rheumatol 1995;24:238-42. 
85 D'Agostino MAet al. EULAR report on the use of ultrasonography in painful knee osteoarthritis. Part 1: prevalence of inflammation in osteoarthritis. Ann Rheum Dis 2005;64:1703-9.

86 Hayashi D, Roemer FW, Katur A, et al. Imaging of synovitis in osteoarthritis: current status and outlook. Semin Arthritis Rheum 2011;41:116-30.

87 Karim Z, Wakefield RJ, Quinn M, et al. Validation and reproducibility of ultrasonography in the detection of synovitis in the knee: a comparison with arthroscopy and clinical examination. Arthritis Rheum 2004;50:387-94.

88 Sarmanova A, Hall M, Fernandes GS, et al. Association between ultrasound-detected synovitis and knee pain: a population-based case-control study with both cross-sectional and follow-up data. Arthritis Res Ther 2017;19.

89 Sarmanova A, Hall M, Moses J, et al. Synovial changes detected by ultrasound in people with knee osteoarthritis - a metaanalysis of observational studies. Osteoarthritis and Cartilage 2016;24:1376-83.

90 Franchi MV, Longo S, Mallinson J, et al. Muscle thickness correlates to muscle cross-sectional area in the assessment of strength training-induced hypertrophy. Scand J Med Sci Sports 2017.

91 Strasser EM, Draskovits T, Praschak M, et al. Association between ultrasound measurements of muscle thickness, pennation angle, echogenicity and skeletal muscle strength in the elderly. Age 2013;35:2377-88.

92 Tan J, Balci N, Sepici V, et al. Isokinetic and isometric strength in osteoarthrosis of the knee. A comparative study with healthy women. Am J Phys Med Rehabil 1995;74:364-9.
93 Berry PA, Jones SW, Cicuttini FM, et al. Temporal relationship between serum adipokines, biomarkers of bone and cartilage turnover, and cartilage volume loss in a population with clinical knee osteoarthritis. Arthritis Rheum 2011;63:700-7.

94 Richette P, Poitou C, Garnero P, et al. Benefits of massive weight loss on symptoms, systemic inflammation and cartilage turnover in obese patients with knee osteoarthritis. Ann Rheum Dis 2011;70:139-44.

95 Excellence NIfHaC. Osteoarthritis: care and management, 2014. Available: https://www.nice.org.uk/guidance/cg177/chapter/1Recommendations\#non-pharmacological-management-2

96 Campbell R, Evans M, Tucker M, et al. Why don't patients do their exercises? Understanding non-compliance with physiotherapy in patients with osteoarthritis of the knee. J Epidemiol Community Health 2001:55:132-8.

97 Jack K, McLean SM, Moffett JK, et al. Barriers to treatment adherence in physiotherapy outpatient clinics: a systematic review. Man Ther 2010;15:220-8.

98 Marks R, Allegrante JP. Chronic osteoarthritis and adherence to exercise: a review of the literature. J Aging Phys Act 2005;13:434-60.

99 Eytan T, Benabio J, Golla V. Social media and the health system. Perm J 2011;15:71-4.

100 Moorhead SA, Hazlett DE, Harrison L, et al. A new dimension of health care: systematic review of the uses, benefits, and limitations of social media for health communication. J Med Internet Res 2013;15:e85.

101 Thackeray R, Crookston BT, West JH. Correlates of health-related social media use among adults. J Med Internet Res 2013;15:e21. 